\title{
Pulmonary valve replacement in primary repair of tetralogy of Fallot in adult patients
}

\author{
Huan Liu ${ }^{1 \#}$, Shun Liu ${ }^{1 \#}$, Anthony Zaki ${ }^{2}$, Xiuwen Wang ${ }^{1}$, Kai Zhu ${ }^{3}$, Yuntao Lu ${ }^{1}$, Ye Yang ${ }^{1}$, Rafi Hamidi ${ }^{1}$, \\ Lai Wei ${ }^{1}$, Chunsheng Wang ${ }^{1}$ \\ ${ }^{1}$ Department of Cardiovascular Surgery, Zhongshan Hospital, Shanghai Cardiovascular Institution, Fudan University, Shanghai, China; ${ }^{2}$ Department \\ of Thoracic and Cardiovascular Surgery, Cleveland Clinic, Cleveland, OH, USA; ${ }^{3}$ School of Clinical Medicine, Jiujiang University, Jiujiang, China \\ Contributions: (I) Conception and design: All authors; (II) Administrative support: C Wang; (III) Provision of study materials or patients: C Wang; (IV) \\ Collection and assembly of data: H Liu, S Liu, X Wang; (V) Data analysis and interpretation: H Liu, K Zhu, Y Yang; (VI) Manuscript writing: All \\ authors; (VII) Final approval of manuscript: All authors. \\ \#These authors contributed equally to this work. \\ Correspondence to: Chunsheng Wang. Fudan University, 1609, Xietu Road, Room 1509, Building 16, Shanghai, China. Email: wcs_heartsurgeon@126.com.
}

Background: Adults with unrepaired tetralogy of Fallot (ToF) are common in developing countries. Longterm overload of the right ventricle places adult patients at risk for postoperative right heart failure after primary repair, which contributes to morbidity and mortality. The effect of pulmonary valve replacement (PVR) in reducing postoperative morbidity and mortality in adults has never been validated.

Methods: We conducted a retrospective cohort study in adults (age $\geq 18$ years) with ToF undergoing primary repair from January 2014 to December 2019 at our institution. Patients were divided into three groups according to techniques used to enlarge the right ventricle outflow tract (RVOT). Baseline variables and perioperative outcomes were collected. The primary endpoint was operative mortality. Secondary endpoints were incidences of right heart failure and stage 3 acute kidney injury (AKI).

Results: A total of 56 patients were enrolled (mean age $41.5 \pm 11.7$ years, 30 females, 53.6\%). They were divided into three groups designated as the following: TA-PVR group for trans-annular patch enlargement with PVR; TA group for trans-annulus patch enlargement without PVR; and group AP for annulus preservation. Four patients $(7.1 \%)$ died postoperatively, all due to right heart failure. All twelve patients in the TA-PVR group survived. There was no significant difference in mortalities among groups. Ten patients (17.9\%) developed right heart failure after surgery with no significant difference among groups. Three patients $(5.4 \%)$ developed stage 3 AKI after surgery, none belonging to the TA-PVR group, however, not statistically significant.

Conclusions: Right heart failure is a common complication after primary repair of adult ToF. Transannulus patch enlargement should be cautiously selected in this population. PVR with trans-annulus patch enlargement may be a promising technique to protect against postoperative right heart failure and mortality when annulus preservation is not feasible.

Keywords: Tetralogy of Fallot (ToF); pulmonary valve replacement; adult congenital heart disease; right heart failure

Submitted Mar 25, 2020. Accepted for publication Jul 22, 2020.

doi: $10.21037 /$ jtd-20-1475

View this article at: http://dx.doi.org/10.21037/jtd-20-1475 


\section{Introduction}

Pulmonary regurgitation (PR) is a common sequela after relief of right ventricular outflow tract (RVOT) obstruction in tetralogy of Fallot (ToF). Several techniques including pulmonary valvotomy, trans-annular patch and incision in the right ventricular (RV) infundibulum predispose to the development of late-onset PR $(1,2)$. Although it may be well tolerated for decades, PR in some patients leads to $\mathrm{RV}$ remodeling, progressive $\mathrm{RV}$ dysfunction and even fatal arrhythmias (3-6). 36-40\% of patients with repaired ToF require reoperation to replace the pulmonary valve within 30 years after primary repair (7). Pulmonary valve replacement (PVR) has been proven to be safe and effective to improve symptoms secondary to chronic $\mathrm{PR}$ in repaired ToF. In the latest American College of Cardiology/American Heart Association guideline, PVR was recommended in symptomatic patients with PR in repaired ToF (Class I) as well as asymptomatic patients with documented RV remodeling (Class IIa) (8).

Adults presenting with unrepaired ToF are rare in Western countries. Limited data exists in English literature regarding the impact of PVR on outcomes after primary surgery for unrepaired adults with ToF. However, patients who have never had surgery for mild ToF during childhood may present as adults with a variety of symptoms and require corrective surgery. In developing countries, adults with unrepaired ToF are not uncommon. It is critical to clarify the impact of PVR on the postoperative outcomes in this unique population.

Operative mortality after repair of adult ToF is higher than in pediatric patients (9). The long-term preoperative $\mathrm{RV}$ pressure overload and the acute volume overload after patch enlargement contributes to right heart failure and low cardiac output in adult ToF. In a study from Brazil, with a cohort consisting of over $80 \%$ of unrepaired adult ToF, the incidence of postoperative low cardiac output was $10.34 \%$ to $14.28 \%$ in patch repair groups (9). The PR after patch enlargement contributed to the high morbidity in this setting.

It is well understood that PVR for repaired ToF in adulthood can reduce the preload of the RV, improve RV dysfunction and decrease the incidence of arrhythmias (10). Studies suggest that the older the age during the primary repair, the higher risk of late PR (7). Consistently, PVR in the primary repair of adult ToF is necessary. However, the effect of PVR with patch enlargement in the primary surgery for adults is unclear. We hypothesized that PVR may reduce postoperative morbidity and mortality in primary surgery for adults with ToF. To our best knowledge, no study on this topic has been conducted.

We present the following article in accordance with the Strengthening the Reporting of Observational Studies in Epidemiology (STROBE) reporting checklist (Available at http://dx.doi.org/10.21037/jtd-20-1475).

\section{Methods}

\section{Patient selection}

A retrospective cohort study was conducted. Patients 18 years or older with the diagnosis of unrepaired ToF, who underwent primary surgery at our institution from January 2009 to December 2019 were enrolled. Exclusion criteria included: previous palliative or corrective repair or other types of conventional heart surgery, endocarditis involving the pulmonary valve, and history of chronic kidney disease.

\section{Study endpoints and definitions}

The primary study objective was to determine whether PVR could reduce the surgical risk in adults undergoing primary repair of ToF. The primary endpoint was operative mortality, and the secondary endpoints were incidence of right heart failure and stage 3 acute kidney injury (AKI). The operative mortality was defined as all-cause mortality during the same admission or within 30 days after index surgery. Right heart failure was defined as central venous pressure $(\mathrm{CVP}) \geq 18 \mathrm{mmHg}$ and cardiac index $(\mathrm{CI})<2.0 \mathrm{~L} / \mathrm{m}^{2}$ for six consecutive hours. AKI was diagnosed and staged based on acute alterations in serum creatinine or urine output according to Acute Kidney Injury Network criteria (11). Prolonged ventilation was defined as requiring continuous mechanical ventilation for more than 120 hours. The demographics and baseline characteristics were compared among groups as well.

\section{Surgical technique}

Operations were performed through midline sternotomy under general anesthesia and with cardiopulmonary bypass. Glutaraldehyde-fixed autologous pericardium or bovine pericardial patch was used to repair the ventricular septal defect (VSD) and enlarge the RVOT. Cardiopulmonary bypass was established through ascending aorta and bicaval cannulations. Myocardial protection was carried out 
with repeated anterograde perfusions with a modified St. Thomas solution every 20 minutes.

Both right atriotomy and pulmonary arteriotomy were made. Through these incisions, the VSD, pulmonary valve, RVOT and other concomitant pathologies were thoroughly inspected. The VSD was repaired through tricuspid valve orifice. The size of pulmonary annulus was deemed acceptable when a $18 \mathrm{~mm}$ Hegar dilator could be passed easily, allowing for an annulus preservation (AP) technique. An isolated RVOT incision was made to resect the infundibular myocardium and to facilitate RVOT patchenlargement. Commissurotomy was performed in case of pulmonary valve stenosis. When the annulus was stenotic, a trans-annulus patch enlargement with or without PVR was performed at surgeon's discretion (TA-PVR or TA). The pulmonary arteriotomy was elongated proximally to widen the annulus and the RVOT. Special caution was made to avoid injury to an abnormal conal branch originating from the right coronary artery. Infundibular myocardium resection was performed to release the subpulmonic narrowing. A patch was used to enlarge the pulmonary annulus and RVOT. Native pulmonary valve was either preserved or replaced with a prosthetic valve. In PVR, the posterior aspect of the sewing ring was sutured to the native annulus, while the anterior aspect was sutured to the corresponding level on the trans-annular patch. It was essential to ensure sutures were placed at an appropriate level for coaxiality between the prosthesis and the RVOT to be maintained. The rest of the operation was performed in a routine fashion.

\section{Date collection and ethics}

Data on all perioperative variables was obtained from our cardiovascular surgical database or manually collected through reviewing the electronic medical record. The data was approved for use in research by the Zhongshan Hospital Institutional Review Board in compliance with the Declaration of Helsinki (as revised in 2013), with patient consent waived.

\section{Statistical analysis}

Continuous variables were expressed as mean \pm standard deviation or median (interquartile range). Categorical variables were presented as frequencies and percentages. For comparison of continuous variables, independent sample t-test was applied when the variable distributions were symmetric and a Wilcoxon rank sum test otherwise. For comparison of categorical variables, the $\chi^{2}$ or Fisher exact test (when appropriate) was used. A P value less than 0.05 was considered significant. The statistical package used was SPSS software (Version 22; IBM Corp, New York, NY, USA).

\section{Results}

\section{Demographics and baseline characteristics}

From January 2014 to December 2019, a total of 56 adult patients (aged $\geq 18$ years, mean age $41.5 \pm 11.7$ years, female $30,53.6 \%$ ) underwent primary repair of ToF at our institution. These patients had no history of previous surgical or interventional procedure to palliate or correct their congenital morphology. Preoperatively, all patients had cyanosis, digital clubbing and symptomatic with a NYHA class 2 or 3 . The median value of NT-proBNP was $203 \mathrm{pg} / \mathrm{mL}(87.6,675.9 \mathrm{pg} / \mathrm{mL})$. All patients were divided into three groups by the technique used to enlarge the RVOT with intention-to-treat principle. The three groups were trans-annular patch enlargement with PVR (TA-PVR group), trans-annular patch enlargement without PVR (TA group) and annulus preservation (AP group). The detail of demographics and baseline characteristics in each group are demonstrated in Table 1. These baseline features were basically balanced among groups except the TA group consisted of statistically significant more female patients than the TA-PVR group.

\section{Baseline echocardiography}

The diagnosis of ToF was made with transthoracic echocardiography (TTE) and verified by intraoperative inspection. The diagnostic morphology met all of the following criterions: typical malalignment VSD, overriding of the aorta, crowding of the RVOT with obstruction, and secondary RV hypertrophy. The mean size of VSD was $20.6 \pm 4.5 \mathrm{~mm}$ in diameter. The degree of aortic overriding varied from $30 \%$ to $75 \%$, with 45 patients $(80.4 \%)$ around $50 \%$. The mean RVOT peak pressure gradient was $104.6 \pm 28.1 \mathrm{mmHg}$. Forty-nine patients $(87.5 \%)$ had thickened and immobile pulmonary valves, while the other seven patients $(12.5 \%)$ had pulmonary valves without significant pathology. There was no pulmonary atresia in the entire cohort. The mean thickness of the RV wall was $10.2 \pm 2.6 \mathrm{~mm}$. Appropriate development of bilateral pulmonary arteries was acceptable in all cases. Development 
Table 1 Demographics and baseline characteristics

\begin{tabular}{|c|c|c|c|c|c|}
\hline & TA-PVR $(n=12)$ & TA $(n=29)$ & AP $(n=15)$ & Total $(\mathrm{N}=56)$ & $P$ \\
\hline Age (years) & $42.2 \pm 10.9$ & $40.1 \pm 12.6$ & $43.7 \pm 10.9$ & $41.5 \pm 11.7$ & 0.617 \\
\hline Hypertension & 0 & $3(10.3)$ & 0 & $3(5.4)$ & 0.416 \\
\hline Diabetes & 0 & 0 & 0 & 0 & NA \\
\hline $\mathrm{sCr}(\mu \mathrm{mol} / \mathrm{L})$ & $78.3 \pm 19.6$ & $69.4 \pm 17.0$ & $76.2 \pm 19.7$ & $73.2 \pm 18.4$ & 0.290 \\
\hline GFR (mL/min) & $96.3 \pm 14.5$ & $99.6 \pm 19.0$ & $93.9 \pm 23.0$ & $97.4 \pm 19.2$ & 0.644 \\
\hline NT-proBNP (pg/ml) & $320.5(97.4,1,091.5)$ & $217.8(110.8,472.6)$ & $97.5(54.3,878.8)$ & $203.8(87.6,675.9)$ & 0.385 \\
\hline
\end{tabular}

$\mathrm{sCr}$, serum creatinine; GFR, glomerular filtration rate. ${ }^{\mathrm{a}, \mathrm{b}}$, significant difference between groups.

Table 2 Baseline echocardiography

\begin{tabular}{|c|c|c|c|c|c|}
\hline & TA-PVR $(n=12)$ & TA $(n=29)$ & AP $(n=15)$ & Total $(\mathrm{N}=56)$ & $P$ \\
\hline $\begin{array}{l}\text { RVOT pressure gradient } \\
(\mathrm{mmHg})\end{array}$ & $113.8 \pm 35.0$ & $105.8 \pm 27.8$ & $95.0 \pm 20.6$ & $104.6 \pm 28.1$ & 0.218 \\
\hline $\begin{array}{l}\text { Pulmonary valve } \\
\text { thickened and } \\
\text { immobile }\end{array}$ & $12(100.0)$ & $26(89.7)^{a}$ & $11(73.3)^{b}$ & $49(87.5)$ & 0.041 \\
\hline Thickness of RV (mm) & $10.9 \pm 1.5$ & $10.3 \pm 2.9$ & $9.5 \pm 2.6$ & $10.2 \pm 2.6$ & 0.501 \\
\hline Diameter of PT $(\mathrm{mm})$ & $23.4 \pm 9.3^{\mathrm{a}}$ & $17.7 \pm 5.8^{\mathrm{b}}$ & $22.3 \pm 7.4$ & $20.2 \pm 7.4$ & 0.036 \\
\hline Diameter of LPA (mm) & $20.0 \pm 7.7^{a}$ & $13.0 \pm 4.0^{\mathrm{b}}$ & $15.4 \pm 4.6^{b}$ & $15.2 \pm 5.8$ & 0.002 \\
\hline Diameter of RPA (mm) & $17.3 \pm 7.4$ & $13.5 \pm 4.1$ & $17.5 \pm 6.2$ & $15.4 \pm 5.8$ & 0.051 \\
\hline LVEF (\%) & $64.5 \pm 4.8$ & $65.8 \pm 4.0$ & $65.0 \pm 6.8$ & $65.3 \pm 5.0$ & 0.742 \\
\hline AR more than mild & $2(16.7)$ & $3(10.3)$ & $2(13.3)$ & $7(12.5)$ & 0.871 \\
\hline MR more than mild & 0 & $1(3.4)$ & 0 & $1(1.8)$ & 1.000 \\
\hline TR more than mild & $5(41.7)$ & $9(31.0)$ & 5 (33.3) & $19(33.9)$ & 0.806 \\
\hline PFO/ASD & $3(25.0)$ & $3(10.3)$ & $2(13.3)$ & $8(14.3)$ & 0.467 \\
\hline
\end{tabular}

ASD, atrial septal defect; AR, aortic regurgitation; LPA, left pulmonary artery; LVEDD, left ventricular end-diastolic diameter; LVEF, left ventricular ejection fraction; MR, mitral regurgitation; PFO, patent foramen ovale; PT, pulmonary trunk; RPA, right pulmonary artery; RV, right ventricle; RVOT, right ventricular outflow track; TR, tricuspid regurgitation; VSD, ventricular septal defect. ${ }^{a, b}$, significant difference between groups.

of the pulmonary arteries was evaluated by contrast CT scan when TTE failed to appreciate these structures due to poor acoustic window. Tricuspid valve regurgitation (TR) was not an uncommon finding in this cohort. Nineteen patients had TR graded more than mild. Three patients had concomitant patent ductus arteriosus (PDA) and two patients had subaortic stenosis. The details of the baseline echocardiography were demonstrated in Table 2. 
Table 3 Procedural outcomes

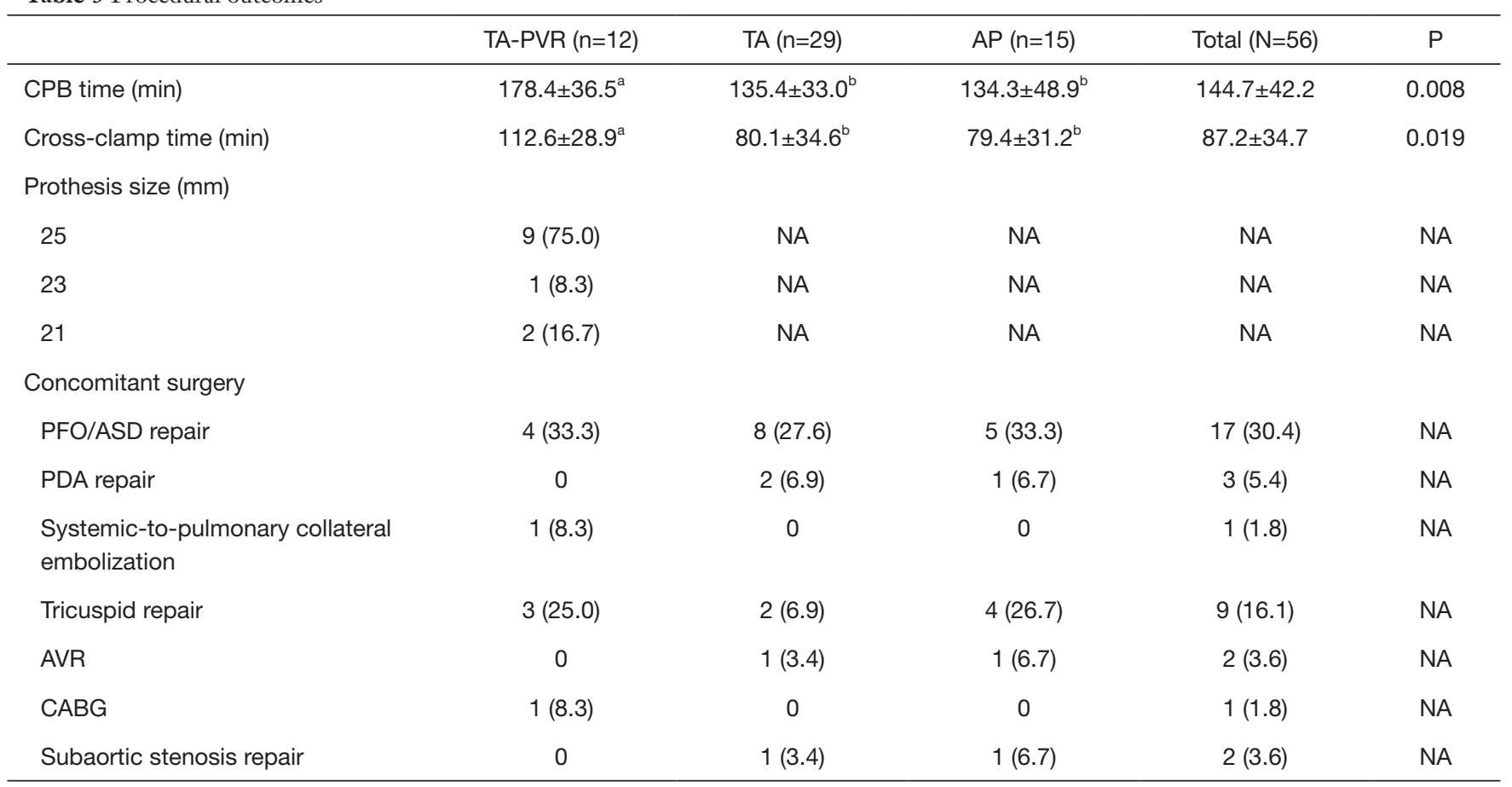

ASD, atrial septal defect; AVR, aortic valve replacement; CABG, coronary artery bypass graft; CPB, cardiopulmonary bypass; PDA, patent ductus arteriosus; PFO, patent foramen ovale. ${ }^{a, b}$, significant difference between groups.

\section{Procedural outcomes}

A total of 56 patients underwent primary ToF repair. The number of patients who underwent trans-annulus enlargement with or without PVR (TA-PVR or TA group) was 12 and 29, respectively. Fifteen patients underwent annulus preservation (AP group). Two patients in TA group developed postoperative right heart failure and were taken back to the operating room for salvage PVR. The PR was deemed to be the cause of RV decompensation in these patients. Bioprosthetic valves were used in all PVR cases.

One patient underwent interventional systemic-topulmonary artery collateral vessel embolization to address hemoptysis prior to the surgery. Seventeen patients underwent patent foramen ovale or atrial septal defect (PFO/ASD) repair. Three patients underwent patent ductus arteriosus (PDA) repair. Nine patients underwent annuloplasty for tricuspid valve repair. Two patients underwent aortic valve replacement, one for aortic valve vegetation due to infectious endocarditis, the other one for bicuspid morphology with moderate regurgitation. One patient with atherosclerotic right coronary stenosis underwent coronary artery bypass grafting with an insitu right internal mammary artery graft. Two patients underwent subaortic stenosis resection. Transesophageal echocardiography (TEE) confirmed the adequacy of surgical correction in all cases. No patient had difficulty weaning from cardiopulmonary bypass. The mean time for aortic cross-clamp and CPB were $87.2 \pm 34.7$ and $144.7 \pm 42.2$ mins, respectively. The cross-clamp and CPB times in the TAPVR group were significantly longer than the other two groups. Hemostasis was achieved easily in all cases. The details of procedural outcomes are demonstrated in Table 3.

\section{Postoperative outcomes}

There were four operative mortalities in this cohort. Three patients in the TA group and one in the AP group died postoperatively. All of these mortalities were contributed to refractory low cardiac output due to right heart failure. All patients in the TA-PVR group survived to hospital discharge, although the difference in operative mortality did not reach a statistical significance among groups. Ten patients $(17.9 \%)$ developed right heart failure without significant difference among groups. Sixteen patients (28.6\%) developed AKI, and three of them required dialysis. No patients developed stage 2 or $3 \mathrm{AKI}$ in the TA- 
Table 4 The postoperative outcomes

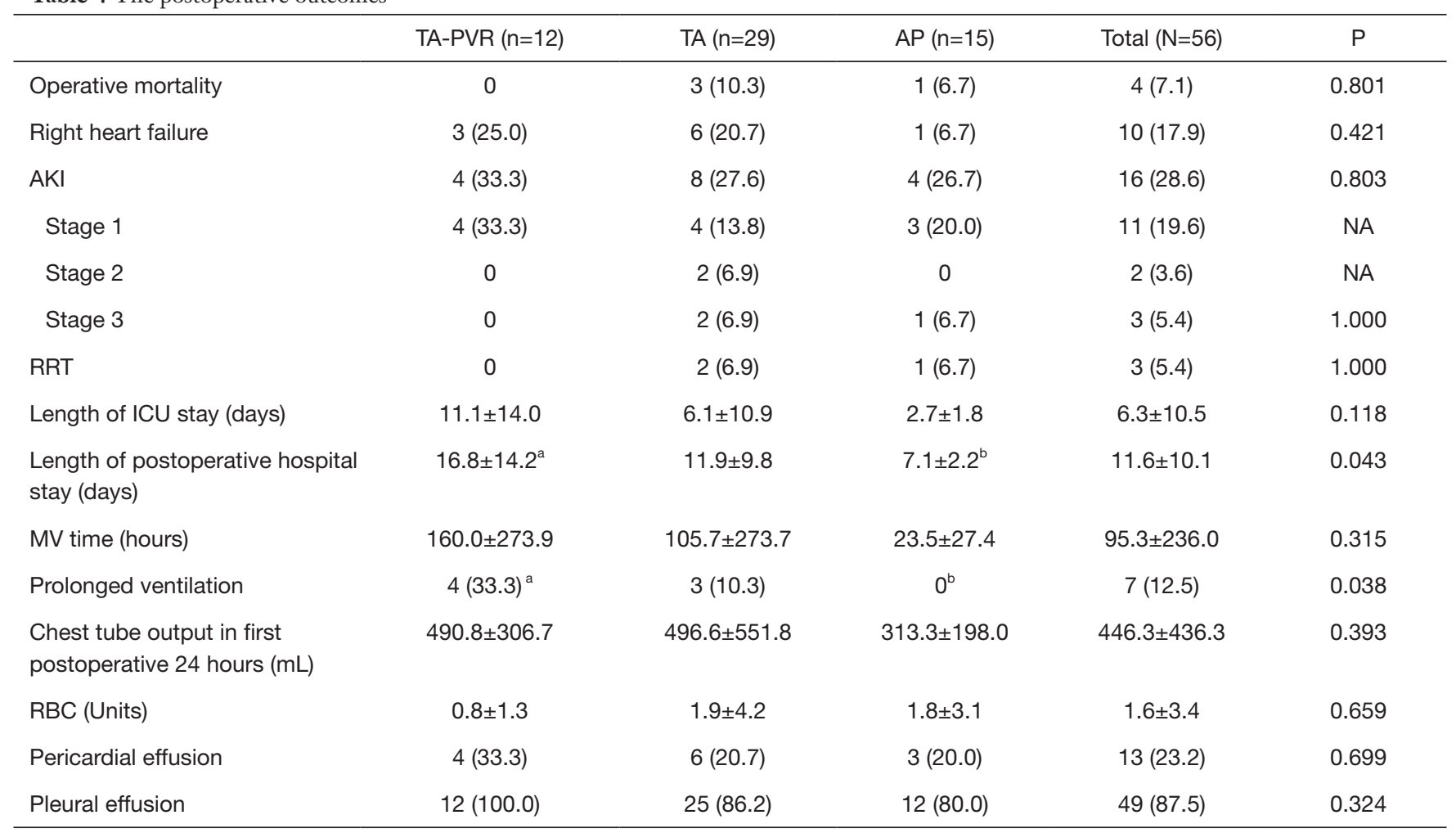

AKI, acute kidney injury; MV, mechanical ventilation; RRT, renal replacement therapy. ${ }^{\mathrm{a}, \mathrm{b}}$, significant difference between groups.

PVR group, although the difference in the incidence of stage 3 AKI did not reach statistical significance among groups. The mean length of stay in the intensive care unit was $6.3 \pm 10.5$ days with no significant difference among groups, however, the length of postoperative hospital stay was significantly longer in the TA-PVR group compared to the AP group $(16.8 \pm 14.2 v s$. $7.1 \pm 2.2$ days). The incidence of prolonged ventilation was significantly higher in the TAPVR group than the AP group (33\% vs. 0). There was no significant difference in blood product transfusion among groups. The details of postoperative outcomes are shown in Table 4.

\section{Discussion}

In Western countries, the majority of adults with ToF have undergone palliative or corrective surgery during childhood. Yet the occasional patients reach adulthood without prior diagnosis or surgical repair; these patients may or may not be symptomatic when they were referred. Different from Western countries, adults with ToF without prior diagnosis are not uncommon in China.
Trans-annulus patch enlargement is often required in adult ToF. Based on the experience of ToF repair in pediatric population, $\mathrm{PR}$ is a common postoperative sequela, which leads to long-term RV dysfunction (12). Surgical or interventional PVR is often required after patients grow into adulthood. The effect of PVR in protection of the RV function has been verified in adult patients with previous repaired $\mathrm{ToF}(13)$.

However, adults undergoing primary repair have different features from the pediatric patients. The afterload of the RV is elevated for an extended time period, which results in a thickened and stiff RV wall. The RV is deemed less compliant and more sensitive to volume overload, which is inevitable when the annulus is patch-enlarged. This has been our experience in dealing with these patients. Right heart failure is a common and fatal complication after ToF repair in adults. In adults, the enlarged RVOT is capable of accommodating a large valve prosthesis. Concomitant PVR in a patch enlargement operation may have a potential benefit of protecting against postoperative right heart failure in adults. We conducted this study to systematically investigate the impact of PVR on postoperative outcomes 
While the technique for PVR is straight-forward, caution has to be paid to determine the appropriate level on the RVOT patch to hold the sutures for the prosthesis. When the sutures are too proximal on the patch, the prosthesis tilts with the orifice toward the patch, resulting in a high residual RVOT pressure gradient. Experienced surgeons estimate the configuration of the patch after the $\mathrm{RV}$ is pressurized to ensure coaxiality between the prosthesis and the RVOT. Beyond that, the size of the patch should be large enough to accommodate at least a $23 \mathrm{~mm}$ prosthesis to reduce RV afterload as much as possible. When an autologous pericardium patch is used, we recommend harvesting the entire area enclosed by the bilateral pleural membrane flexion, left brachiocephalic vein and the diaphragm. Not surprisingly, PVR prolongs myocardial ischemia and cardiopulmonary times by at least 30 minutes.

The demographics and baseline characteristics were balanced among groups. However, we noticed a significant difference in proportion of female patients between the TA-PVR and the TA group. This might be explained by the potential fertility requirements from the females, which promoted the surgeons to avoid valve replacement. However, we believe that the gender difference has little influence on the postoperative outcomes.

Twenty-six patients $(89.7 \%)$ in the TA group had thickened and immobile pulmonary valves, significantly higher than the AP group (11 patients, 73.3\%). All patients in the TA-PVR group had these features as well, however, no significant difference was reached due to low case number. Patients in the AP group generally had normal sized pulmonary annulus. The association between small pulmonary annulus and pulmonary valve dysplasia has been reported in previous study (14). There were some differences in the size of pulmonary trunk, left pulmonary artery and left ventricular end-diastolic diameter (LVEDD) among these groups, which might be a result from the gender difference, however, these values were all within normal ranges.

The results of freedom from prosthesis deterioration and reoperation in previous studies on PVR with tissue valves are encouraging $(15,16)$. Although, the long-term outcomes after mechanical valve replacement in pulmonary valve position are also promising (17), the patients undergoing tissue valve replacement can take the advantage of avoiding lifetime anticoagulation therapy and the feasibility of "valvein-valve" technique to treat valve deterioration. As patients in this cohort were relatively young, a postoperative active lifestyle was critical. After the pros and cons were discussed with the patients, tissue valves were implanted in all TAPVR cases.

Nine patients $(75 \%)$ in the TA-PVR group received a $25 \mathrm{~mm}$. In previous publications, the size of the prosthesis was a protective factor against reintervention, and $25 \mathrm{~mm}$ or greater prosthesis were used in most of the patients in these studies $(18,19)$. Technically, when the RVOT is patchenlarged, there should be minimal difficulty in implanting at least a $25 \mathrm{~mm}$ prosthesis in an adult habitus. We had two patients with $21 \mathrm{~mm}$ prosthesis in this cohort, however, they were both female with small habitus.

The number of PFO or ASD diagnosed by preoperative echocardiography was less than the number identified during intraoperative inspection. This indicates that $\mathrm{PFO}$ or ASD can be easily misdiagnosed by preoperative TTE. A thorough inspection is mandatory to avoid missing any uncorrected pathology.

One patient underwent systemic-to-pulmonary collateral embolization to treat hemoptysis prior to the surgical correction. Hemoptysis is an uncommon complication in patients with cyanotic congenital heart disease (20). It is the result of rupture of major aortopulmonary collateral arteries (MAPCAs), which can be treated effectively by coil embolization (21). The case in our study did not experience any reoccurrence during hospital stay and follow-up.

The operative mortality of the entire cohort was $7.1 \%$ (four patients), which was consistent with previous study (22). All 12 patients in the TA-PVR group survived to hospital discharge. However, there was no significant difference in mortality among groups, which might be a result of low case number. Three patients died in the TA group. Two of them developed postoperative right heart failure which was deemed related to PR. They were taken back to the operating room for salvage PVR. Unfortunately, the hemodynamics of these patients continued to deteriorate and died shortly after the reintervention. It indicates that early replacement of the pulmonary valve may protect the $\mathrm{RV}$ against decompensated right heart failure. We conclude that following surgical correction, the requirement of moderate or higher dosage of inotropics or difficulty weaning from cardiopulmonary bypass should be considered as an indication of PVR.

No patient required renal replacement therapy in the TA-PVR group. The increased right heart filling pressure has been identified as an independent risk factor for impaired renal function in patients with heart failure (23). PVR can eliminate the postoperative PR and reduce RV preload. As a result, we believe that PVR has a protective 
role against $\mathrm{AKI}$ in this setting.

A trend of shorter recovery period was observed in the AP group, including shorter length of ICU stay and mechanical ventilation time, although not statistically significant. The patients in the TA-PVR group had significantly longer length of postoperative hospital stay and higher incidence of prolonged ventilation than the AP group. The difference might be contributed to the heterogeneity between these two groups. Patients in the AP group were unlikely to have a dysplastic RV infundibulum and experienced preserved RV function than patients with small annulus (14).

However, the difference in early outcomes between trans-annular patch and annulus preservation technique is not obvious in the pediatric population (24). We believe that the long-term elevation of RV afterload caused by the annulus dysplasia makes the RV thickened and less compliant in adults. The RV is more sensitive to volume overload and prone to fail. As a result, the trans-annulus enlargement should be cautiously selected in adult patients. The annulus should be preserved as much as possible with a diameter greater than $17 \mathrm{~mm}$.

The nature of this study is descriptive. As such, our surgical series of 56 patients may not provide adequate power for robust statistical comparisons between the groups as defined in this study. It would be desirable to evaluate larger samples of patients from multiple institutions in order to determine whether our findings are generalizable. In addition, we are interested in the late outcomes regarding RV function and prosthesis-related complications. Future studies with longer follow-up are planned.

\section{Conclusions}

Right heart failure is a common and fatal complication after primary repair of adult ToF. Trans-annulus patch enlargement should be cautiously selected in this population. PVR in trans-annulus patch enlargement may be a promising technique to protect against postoperative acute renal failure and mortality.

\section{Acknowledgments}

Funding: None.

\section{Footnote}

Reporting Checklist: The authors have completed the
Strengthening the Reporting of Observational Studies in Epidemiology (STROBE) reporting checklist. Available at http://dx.doi.org/10.21037/jtd-20-1475

Data Sharing Statement: Available at http://dx.doi. org/10.21037/jtd-20-1475

Conflicts of Interest: All authors have completed the ICMJE uniform disclosure form (available at http://dx.doi. org/10.21037/jtd-20-1475). The authors have no conflicts of interest to declare.

Ethical Statement: The authors are accountable for all aspects of the work in ensuring that questions related to the accuracy or integrity of any part of the work are appropriately investigated and resolved. The data was approved for use in research by the Zhongshan Hospital Institutional Review Board in compliance with the Declaration of Helsinki (as revised in 2013), with patient consent waived.

Open Access Statement: This is an Open Access article distributed in accordance with the Creative Commons Attribution-NonCommercial-NoDerivs 4.0 International License (CC BY-NC-ND 4.0), which permits the noncommercial replication and distribution of the article with the strict proviso that no changes or edits are made and the original work is properly cited (including links to both the formal publication through the relevant DOI and the license). See: https://creativecommons.org/licenses/by-nc-nd/4.0/.

\section{References}

1. Kirklin JK, Kirklin JW, Blackstone EH, et al. Effect of transannular patching on outcome after repair of tetralogy of Fallot. Ann Thorac Surg 1989;48:783-91.

2. d'Udekem d'Acoz Y, Pasquet A, Lebreux L, et al. Does right ventricular outflow tract damage play a role in the genesis of late right ventricular dilatation after tetralogy of Fallot repair? Ann Thorac Surg 2003;76:555-61; discussion 561.

3. Geva T. Repaired tetralogy of Fallot: the roles of cardiovascular magnetic resonance in evaluating pathophysiology and for pulmonary valve replacement decision support. J Cardiovasc Magn Reson 2011;13:9.

4. Geva T, Sandweiss BM, Gauvreau K, et al. Factors associated with impaired clinical status in longterm survivors of tetralogy of Fallot repair evaluated 
by magnetic resonance imaging. J Am Coll Cardiol 2004;43:1068-74.

5. Harrild DM, Berul CI, Cecchin F, et al. Pulmonary valve replacement in tetralogy of Fallot: impact on survival and ventricular tachycardia. Circulation 2009;119:445-51.

6. Frigiola A, Redington AN, Cullen S, et al. Pulmonary regurgitation is an important determinant of right ventricular contractile dysfunction in patients with surgically repaired tetralogy of Fallot. Circulation 2004;110:III153-7.

7. Hickey EJ, Veldtman G, Bradley TJ, et al. Late risk of outcomes for adults with repaired tetralogy of Fallot from an inception cohort spanning four decades. Eur J Cardiothorac Surg 2009;35:156-64; discussion 164.

8. Stout KK, Daniels CJ, Aboulhosn JA, et al. 2018 AHA/ ACC Guideline for the Management of Adults With Congenital Heart Disease: A Report of the American College of Cardiology/American Heart Association Task Force on Clinical Practice Guidelines. Circulation 2019;139:e698-e800.

9. Khan I, Tufail Z, Afridi S, et al. Surgery for Tetralogy of Fallot in Adults: Early Outcomes. Braz J Cardiovasc Surg 2016;31:300-3.

10. Ferraz Cavalcanti PE, Sa MP, Santos CA, et al. Pulmonary valve replacement after operative repair of tetralogy of Fallot: meta-analysis and meta-regression of 3,118 patients from 48 studies. J Am Coll Cardiol 2013;62:2227-43.

11. Mehta RL, Kellum JA, Shah SV, et al. Acute Kidney Injury Network: report of an initiative to improve outcomes in acute kidney injury. Crit Care 2007;11:R31.

12. Ammash NM, Dearani JA, Burkhart HM, et al. Pulmonary regurgitation after tetralogy of Fallot repair: clinical features, sequelae, and timing of pulmonary valve replacement. Congenit Heart Dis 2007;2:386-403.

13. Heng EL, Gatzoulis MA, Uebing A, et al. Immediate and Midterm Cardiac Remodeling After Surgical Pulmonary Valve Replacement in Adults With Repaired Tetralogy of Fallot: A Prospective Cardiovascular Magnetic Resonance and Clinical Study. Circulation 2017;136:1703-13.

14. Chacko BR, Chiramel GK, Vimala LR, et al. Spectrum of pulmonary valve morphology and its relationship to

Cite this article as: Liu H, Liu S, Zaki A, Wang X, Zhu K, Lu Y, Yang Y, Hamidi R, Wei L, Wang C. Pulmonary valve replacement in primary repair of tetralogy of Fallot in adult patients. J Thorac Dis 2020;12(9):4833-4841. doi: 10.21037/jtd-201475 pulmonary trunk in tetralogy of Fallot. Indian J Radiol Imaging 2017;27:65-9.

15. Batlivala SP, Emani S, Mayer JE, et al. Pulmonary valve replacement function in adolescents: a comparison of bioprosthetic valves and homograft conduits. Ann Thorac Surg 2012;93:2007-16.

16. Jang W, Kim YJ, Choi K, et al. Mid-term results of bioprosthetic pulmonary valve replacement in pulmonary regurgitation after tetralogy of Fallot repair. Eur J Cardiothorac Surg 2012;42:e1-8.

17. Pragt H, van Melle JP, Javadikasgari H, et al. Mechanical valves in the pulmonary position: An international retrospective analysis. J Thorac Cardiovasc Surg 2017;154:1371-1378 e1.

18. Nomoto R, Sleeper LA, Borisuk MJ, et al. Outcome and performance of bioprosthetic pulmonary valve replacement in patients with congenital heart disease. J Thorac Cardiovasc Surg 2016;152:1333-1342 e3.

19. Chen PC, Sager MS, Zurakowski D, et al. Younger age and valve oversizing are predictors of structural valve deterioration after pulmonary valve replacement in patients with tetralogy of Fallot. J Thorac Cardiovasc Surg 2012;143:352-60.

20. Haroutunian LM, Neill CA. Pulmonary complications of congenital heart disease: hemoptysis. Am Heart J 1972;84:540-59.

21. van der Weijden P, Baur LH, Kool LJ, et al. Embolization as a treatment of life-threatening haemoptysis in an adult with Tetralogy of Fallot with pulmonary atresia. Int J Card Imaging 1998;14:123-6.

22. Attenhofer Jost CH, Connolly HM, Burkhart HM, et al. Tetralogy of fallot repair in patients 40 years or older. Mayo Clin Proc 2010;85:1090-4.

23. Konstam MA, Kiernan MS, Bernstein D, et al. Evaluation and Management of Right-Sided Heart Failure: A Scientific Statement From the American Heart Association. Circulation 2018;137:e578-e622.

24. Ismail SR, Kabbani MS, Najm HK, et al. Early outcome of tetralogy of Fallot repair in the current era of management. J Saudi Heart Assoc 2010;22:55-9. 